\title{
UNA REVISIÓN NARRATIVA SOBRE EL PENSAMIENTO COMPUTACIONAL EN EDUCACIÓN SECUNDARIA OBLIGATORIA
}

\author{
Óscar Marañón Marañón \\ Higinio González-García \\ Universidad Internacional de La Rioja (UNIR)
}

\begin{abstract}
RESUMEN: El objetivo de este trabajo fue conocer los beneficios que el pensamiento computacional aporta a la educación y las propuestas de aplicación del mismo en Educación Secundaria tanto en las distintas regiones españolas como en otros países europeos. Para ello se ha realizado una revisión narrativa de la literatura científica, extrayéndose artículos tanto en castellano como en inglés de las siguientes bases de datos: Dialnet, Scopus, Google Scholar, Researchgate y Microsoft Academic. Entre los principales resultados hallados destacan la tendencia actual en muchos países a introducirlo en el currículo, así como una serie de habilidades, características y actitudes asociadas a él, entre las que destacan la abstracción, la descomposición de problemas, el pensamiento algorítmico, la automatización, la depuración de errores y la generalización. Entre los principales beneficios que aporta el pensamiento computacional destacan: mejora de la competencia digital, desarrollo y fortalecimiento de habilidades socio-emocionales, habilidades relacionadas con el lenguaje y la comunicación y potenciación del trabajo colectivo. Como conclusión final es importante destacar que la mayoría de los países Europeos ha implementado o está en vías de implementar más acciones para fortalecer el uso del pensamiento computacional entre el alumnado.
\end{abstract}

PALABRAS CLAVE: pensamiento abstracto, competencia digital, educación secundaria obligatoria.

\section{A NARRATIVE REVIEW OF COMPUTATIONAL THINKING IN COMPULSORY SECONDARY EDUCATION}

\footnotetext{
ABSTRACT: The objective of this work was to know the benefits that computational thinking brings to education and the proposals for its application in Secondary Education in the different Spanish regions and other European countries. For this, a narrative review of the scientific literature has
} 
been carried out, extracting articles in both languages Spanish and English from the following databases: Dialnet, Scopus, Google Scholar, Researchgate and Microsoft Academic. Among the main results found, in many countries the current trend is to introduce it into the curriculum, as well as a series of skills, characteristics and attitudes associated with it, including abstraction, problem decomposition, algorithmic thinking, automation, debugging and generalization. Among the main benefits that computational thinking brings is the improvement of digital competence, development and strengthening of socio-emotional skills, skills related to language and communication, and empowerment of collective work. As a conclusion, it is important to highlight that most of the European countries have implemented or are in the process of implementing more actions to strengthen the use of computational thinking among students.

KEYWORDS: Abstract thinking, digital skill, secondary compulsory education.

Recibido: 28/04/2020

Aceptado: 21/10/2020

Correspondencia: Higinio González-García, Facultad de Educación, Universidad Internacional de La Rioja (UNIR), Calle Almansa 101, 28040 Madrid. Email: higinio. gonzalez@unir.net

\section{INTRODUCCIÓN}

Existe una tendencia europea y mundial a introducir el pensamiento computacional en el currículo, en este sentido 16 países europeos ya han incluido el pensamiento computacional en sus centros educativos (Bocconi, Chioccariello, Dettori, Ferrari y Engelhardt, 2016; Roig-Vila y Moreno-Isac, 2020). Sin embargo, en España la introducción del pensamiento computacional varía de unas Comunidades Autónomas a otras. De este modo, en Galicia, Cataluña, Castilla-La Mancha, Castilla y León, Comunidad Valenciana, Murcia y Andalucía, existen iniciativas para la introducción del pensamiento computacional tanto en primaria como en secundaria (INTEF, 2018; Roig-Vila y Moreno-Isac, 2020). Además, la tendencia es incluir el pensamiento computacional en materias relacionadas con la tecnología y la informática, por lo que es probable que los alumnos que no cursen estas materias no tengan acceso a los beneficios que el pensamiento computacional puede aportarles (Bocconi et al., 2016; Caballero-González y García-Valcárcel, 2019; INTEF, 2018).

Como principal beneficio, el Pensamiento Computacional permite a los jóvenes pensar de una forma distinta cuando se trata de resolver problemas (Lee, Martin, Denner, Coulter, Allan, Erickson, Malyn-Smith y Werner, 2011), desarrollando la capacidad de descubrir, crear e innovar (Allan, Coulter, Denner, Erickson, Lee, Malyn-Smith y Martin, 2010), o comprender qué puede ofrecerles la tecnología. De esta forma, el 
pensamiento computacional puede ayudar a los alumnos a superar las dificultades de comprensión de los conceptos abstractos de las materias del currículum de secundaria (Sánchez, 2018). En ese sentido, con frecuencia se relaciona el pensamiento computacional con la programación y la informática (Basogain, Olabe y Olabe, 2015; INTEF, 2018), lo cual es uno de los errores más habituales, ya que, el pensamiento computacional es aplicable a múltiples disciplinas (Zapata-Ross, 2015). Además, el pensamiento computacional permite a los alumnos expresarse a través de medios digitales, favoreciendo las habilidades sociales y la competencia lingüística. Asimismo, permite mejorar la competencia del sentido de la iniciativa y espíritu emprendedor, ya que fomenta el liderazgo, la innovación y el emprendimiento (Posada, 2017).

En cuanto al origen del término pensamiento computacional (computational thinking), fue definido por primera vez por Wing (2006) en la publicación Comunicaciones de la Association for Computing Machinery: "El Pensamiento Computacional implica resolver problemas, diseñar sistemas y comprender el comportamiento humano, basándose en los conceptos fundamentales de la ciencia de la Computación. El Pensamiento Computacional incluye una amplia variedad de herramientas mentales que reflejan la amplitud del campo de la Computación". (p. 33)

Este artículo supuso el inicio de un debate internacional sobre el valor del pensamiento computacional para la educación. A pesar de que la definición propuesta por esta autora fue la primera y goza de amplia aceptación, no hay un consenso internacional sobre la definición de pensamiento computacional (Román, Pérez y Jiménez, 2015). Posteriormente, Wing (2011) propuso una nueva definición de pensamiento computacional, "El Pensamiento Computacional son los procesos de pensamiento implicados en la formulación de problemas y sus soluciones para que estas últimas estén representadas de forma que puedan llevarse a cabo de manera efectiva por un procesador de información" (p. 1).

Aunque la definición de Wing es un referente en la comunidad internacional, existen otras definiciones de pensamiento computacional. Organizaciones como Computer Science Teachers Association y la International Society for Technology in Education (CSTA e ISTE, 2011), así como la Royal Society (Royal Society, 2012) han desarrollado sus propias definiciones. También existen multitud de autores que han propuesto definiciones $\mathrm{o}$, cuando menos, aspectos relacionados con el pensamiento computacional.

Por último, destacar la existencia de dos tendencias principales que justifican la introducción del pensamiento computacional en la enseñanza obligatoria. Por un lado, las habilidades que desarrolla el pensamiento computacional para que los alumnos puedan pensar de manera diferente, resolver problemas del mundo real y analizar temas del día a día, que afecten a los alumnos, desde una perspectiva distinta (Bocconi et al., 2016). Por otro lado, la necesidad de cubrir puestos de trabajo en los sectores TIC y de prepararse para los empleos del futuro, justifican la introducción del pensamiento computacional en la enseñanza obligatoria (Bocconi et al., 2016). Por ello, el objetivo de este trabajo es: conocer las propuestas de aplicación del pensamiento computacional en Educación Secundaria y su evolución en las distintas regiones españolas y otros países europeos. 


\section{Metodología}

El presente trabajo consiste en una revisión narrativa de la literatura científica sobre pensamiento computacional y su introducción en la Enseñanza Secundaria Obligatoria. Para dicho fin se han buscado los artículos existentes en esta área, revisándose únicamente artículos a texto completo redactados en inglés o castellano publicados en revistas especializadas. Se han revisado, asimismo, informes de carácter oficial, tanto a nivel de España como a nivel de la Unión Europea. Las búsquedas fueron de artículos, artículos de revisión, tesis doctorales, que estuvieran en bases de datos de Dialnet, Scopus, Google Scholar, Researchgate y Microsoft Academic, publicados entre 2010 y 2018, aunque de manera excepcional se sacaron dos artículos de las referencias de las búsquedas encontradas (Wing, 2006; Wing, 2008). Las palabras clave utilizadas fueron las siguientes: "pensamiento computacional, educación secundaria", "pensamiento computacional, currículo", "computational thinking, secondary education", "computational thinking, curriculum", realizando la búsqueda en el título, resumen, las palabras clave y el texto completo. Se seleccionaron 27 trabajos de investigación que se ajustaban en mayor medida a las palabras clave. Asimismo, también se revisaron las listas de referencias bibliográficas de los artículos seleccionados. Se excluyeron los estudios que estuvieran publicados con anterioridad a la fecha marcada. En resumen, si se desglosan las características principales de los artículos seleccionados, destacan las siguientes:

- 17 artículos en inglés y 10 artículos en español.

- 10 artículos teóricos sobre el pensamiento computacional.

- 5 experiencias prácticas llevadas a cabo.

- 6 informes que dotan resultados sobre la proyección a las aulas.

- 6 trabajos con referencia explícita a España o sus autonomías.

\section{Habilidades del pensamiento computacional}

Aunque Wing (2006) introdujo por primera vez el término pensamiento computacional, no hay un consenso internacional sobre la definición del mismo. Sin embargo, en la literatura habitualmente se asocia el pensamiento computacional con una serie de habilidades relevantes para su inclusión en la Enseñanza Secundaria Obligatoria. Estas habilidades hacen referencia a la abstracción, descomposición de problemas, pensamiento algorítmico, automatización, depuración y generalización:

- Abstracción. Diversos autores mencionan la abstracción como una de las habilidades del pensamiento computacional (Angeli et al., 2016; Barr y Stephenson, 2011; Grover y Pea, 2013; Lee et al., 2011; Selby y Woollard, 2013; Wing, 2011). En el ámbito de pensamiento computacional, la abstracción consiste en eliminar los detalles irrelevantes de un problema, de tal manera que el problema se vuelve más fácil, pero sin perder lo importante del problema.

- Descomposición de problemas. (Angeli et al., 2016; Barr y Stephenson, 2011; Grover y Pea, 2013; Selby y Woollard, 2013; Wing, 2006) coinciden en men- 
cionar la descomposición de problemas como otra de las habilidades más importantes del pensamiento computacional. La descomposición de problemas hace referencia a una manera de pensar en un problema en términos de sus componentes. Se requiere que se entienda, solucione, desarrolle y evalúe cada componente por separado. De esta forma se facilita la resolución de problemas complejos.

- Pensamiento algorítmico. Los mismos autores que recogen la descomposición de problemas como una de las habilidades del pensamiento computacional, también coinciden en considerar el pensamiento algorítmico como otras de sus habilidades fundamentales (Angeli et al., 2016; Barr y Stephenson, 2011; Grover y Pea, 2013; Selby y Woollard, 2013; Wing, 2011). Consideran el pensamiento algorítmico como una manera de alcanzar una solución a un problema a través de una definición clara de los pasos a llevar a cabo.

- Automatización. Algunos autores señalan la automatización de tareas como una de las habilidades del pensamiento computacional (Barr y Stephenson, 2011; Lee et al., 2011; Wing, 2008).

- Depuración. Angeli et al. (2016) y Grover y Pea (2013) recogen la depuración como otra habilidad del pensamiento computacional. La depuración hace referencia a la corrección de fallos en los pasos definidos para la resolución del problema.

- Generalización. Varios de estos autores también coinciden en señalar la generalización como otra habilidad importante del pensamiento computacional (Angeli et al., 2016; Selby y Woollard, 2013; Wing, 2011). La generalización supone la identificación de patrones en la solución de problemas que permitan ser aplicados en la resolución de nuevos problemas.

Tabla 1. Habilidades del pensamiento computacional según autores.

Fuente: elaboración propia

\begin{tabular}{|c|c|c|c|c|c|c|}
\hline$\frac{\frac{1}{2}}{\frac{3}{2}}$ & $\begin{array}{l}\frac{0}{0} \\
\frac{0}{0} \\
\frac{0}{2} \\
\frac{0}{2}\end{array}$ & 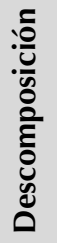 & 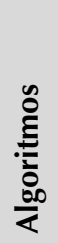 & 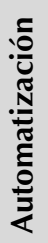 & 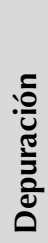 & 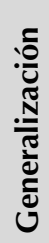 \\
\hline Angeli et al., 2016 & & & & & & \\
\hline Barr y Stephenson, 2011 & & & & & & \\
\hline Grover y Pea, 2013 & & & & & & \\
\hline Lee et al., 2011 & & & & & & \\
\hline Selby y Woollard, 2013 & & & & & & \\
\hline Wing, 2006, 2008, 2011 & & & & & & \\
\hline
\end{tabular}


Dadas las habilidades mencionadas en los párrafos anteriores, podría definirse el pensamiento computacional como un procedimiento de resolución de problemas basado en la abstracción, descomposición, algoritmos, automatización, depuración y generalización. Además de las habilidades mencionadas en la Tabla 1, existen otras habilidades asociadas al pensamiento computacional, aunque no gozan del consenso de las anteriores, como pensamiento iterativo, recursivo y paralelo, paralelización, simulación, análisis, lógica condicional, etc. (Bocconi et al., 2016; Zapata-Ross, 2015; Román, Pérez y Jiménez, 2015).

Al mismo tiempo, el pensamiento computacional no sólo se caracteriza por las habilidades mencionadas anteriormente, sino también por actitudes. A este respecto, varios autores recogen la seguridad ante la complejidad, la persistencia a la hora de enfrentarse a problemas difíciles y la capacidad para enfrentarse a problemas abiertos, como actitudes que fomentan el pensamiento computacional, como son Barr, Harrison y Conery (2011) y Weintrop et al. (2016). Además, es importante señalar que Barr, Harrison y Conery (2011) también recogen otras actitudes, como son la capacidad para gestionar la ambigüedad y la capacidad para comunicarse y trabajar en equipo para alcanzar un objetivo. Por su parte, Woollar (2016) añade la creación, experimentación, perseverancia y colaboración como actitudes y/o cualidades del pensamiento computacional.

A su vez, en la actualidad se usan gran cantidad de términos en relación al pensamiento computacional. A este respecto, uno de los términos que más se relaciona con pensamiento computacional es programación (Basogain, Olabe y Olabe, 2015; INTEF, 2018). A menudo, se entiende que el pensamiento computacional es básicamente programar. Sin embargo, el pensamiento computacional implica mucho más que únicamente programar (Lamprou y Repenning, 2018; Grover, 2018). Así mismo, se relaciona habitualmente con la informática, con la competencia digital, con la resolución de problemas y el pensamiento algorítmico, entre otros (Bocconi, Chioccariello, Dettori, Ferrari y Engelhardt, 2016). A este respecto, los conceptos con los que se vincula al pensamiento computacional son (Bocconi et al., 2016):

- Resolución de problemas

- Programación

- Codificación

- Informática

- Ciencias de la informática

- Tecnologías de la información

- Informática

- Competencia digital

- Pensamiento algorítmico

- Tecnologías de la información 
Por lo tanto, aunque no existe una única definición de pensamiento computacional, sí existen una serie de habilidades, características y actitudes asociadas al pensamiento computacional que gozan de un amplio consenso.

\section{BenfFicios del uso del peNSAMIENTO COMPUTACIONAL EN EDUCACIÓN}

En cuanto a las ventajas del uso del pensamiento computacional, distintos artículos mencionan las ventajas de su uso en las aulas de secundaria. En particular, el pensamiento computacional permite a los alumnos pensar de una manera diferente a la hora de resolver problemas, analizar los temas cotidianos desde una óptica diferente (Lee et al., 2011), desarrollar la capacidad de descubrir, crear e innovar (Allan et al., 2010), o entender lo que la tecnología puede ofrecerles.

El pensamiento computacional permite trabajar con propuestas de problemas reales, que conecten con la realidad de los alumnos y les permite enfrentarse a distintas situaciones sin repetir soluciones prediseñadas, sino que les permite ser creativos a la hora de solucionar problemas (Gurises Unidos, 2017) Además, la conexión con problemas reales mejora el aprendizaje de los discentes. También favorece en los alumnos un mejor uso de la tecnológica, permitiéndoles ser creadores de tecnología y no meros usuarios, lo que estimula su creatividad y les ayuda a prepararse para las nueva realidad social y laboral con la que se van a encontrar. Es decir, mejora la competencia digital, una de las competencias clave (Gurises Unidos, 2017).

Asimismo, el pensamiento computacional, además de mejorar la competencia digital, fomenta otras habilidades y competencias, igualmente importantes para el alumno (Gurises Unidos, 2017):

- Habilidades socio-emocionales: trabajar el pensamiento computacional partiendo de problemas reales que sean desafiantes, permite desarrollar y fortalecer habilidades socio-emocionales: autoconcepto y autoeficacia para enfrentarse al problema, perseverancia en el esfuerzo, así como la comunicación y la empatía para trabajar en grupo.

- Lenguaje y comunicación: en el proceso de resolución de problemas mediante pensamiento computacional, se ponen en práctica habilidades relacionadas con el lenguaje y la comunicación, como la escucha activa, la reflexión, la tolerancia, el respeto a las opiniones de los demás miembros del equipo y la empatía.

- Potenciación del trabajo colectivo: el primer paso en la resolución de un problema mediante pensamiento computacional consiste en la formulación del problema. En esta primera fase es necesaria la aportación de distintos puntos de vista ya que no hay una única manera de resolver un problema. Una vez definido el problema, el siguiente paso es descomponerlo en problemas más pequeños que puedan ser resueltos de manera más sencilla. En esta fase hay un campo fértil para trabajar en equipo.

Asimismo, permite mejorar la competencia del sentido de la iniciativa y espíritu emprendedor, ya que fomenta el liderazgo, la innovación y el emprendimiento (Posada, 2017). Por todo lo anteriormente mencionado, el pensamiento computacional 
contiene características positivas y necesarias de ser fomentadas en el alumnado de Educación Secundaria Obligatoria.

\section{RelACión Del PENSAMIENTO COMPUTACIONAL CON EL CURRíCULO ESPAÑOL}

El currículo de enseñanza secundaria obligatoria viene establecido en España por el Real Decreto 1105/2014, de 26 de diciembre. Este Real Decreto sigue las recomendaciones del Parlamento Europeo y el Consejo sobre las competencias clave para el aprendizaje permanente. En este sentido, propone nuevos enfoques en el aprendizaje y en la evaluación. Se adopta la denominación de las competencias clave definidas por la Unión Europea. Estas competencias se encuentran entre los beneficios atribuidos al pensamiento computacional, tal y como se ha reflejado en el apartado de los beneficios que el pensamiento computacional aporta a la educación. El Real Decreto 1105/2014 establece las competencias del currículo:

- Competencia lingüística.

- Competencia matemática y competencias básicas en ciencia y tecnología.

- Competencia digital.

- Aprender a aprender.

- Competencias sociales y cívicas.

- Sentido de iniciativa y espíritu emprendedor.

- Conciencia y expresiones culturales.

Dadas las habilidades, características y actitudes asociadas al pensamiento computacional (Basogain et al., 2015; Grover, 2018; Lamprou y Repenning, 2018), mediante su introducción en el currículo, se pueden trabajar las siguientes competencias:

- Competencia digital: el pensamiento computacional permite trabajar de manera directa esta competencia mediante el uso de las TIC. Fomenta el uso crítico de las TIC y fomenta que los alumnos pasen de ser consumidores de tecnología a creadores de tecnología.

- Competencia matemática: el pensamiento algorítmico permite desarrollar la competencia matemática.

- Competencia lingüística: en el proceso de resolución de problemas mediante pensamiento computacional, se ponen en práctica habilidades relacionadas con el lenguaje y la comunicación.

- Competencias sociales y cívicas: trabajar el pensamiento computacional partiendo de problemas reales que sean desafiantes, permite desarrollar y fortalecer habilidades socio-emocionales.

- Sentido de iniciativa y espíritu emprendedor. El pensamiento computacional permite a los alumnos desarrollar la capacidad de descubrir, crear e innovar (Allan et al., 2010). 


\section{Tendencias en la integraCión del PeNSAMIENTO COMPUTACIONAL EN LA ENSEÑANZA OBLIGATORIA EN EUROPA}

El informe "Developing Computational Thinking in Compulsory Education - Implications for policy and practice" elaborado en 2017 por la Unión Europea (Bocconi et al., 2016) recoge la situación del pensamiento computacional en el currículo de la enseñanza obligatoria en los países europeos. Tal y como se ha reflejado en el apartado sobre la definición del pensamiento computacional, existen distintas definiciones sobre pensamiento computacional. Por lo tanto, no todos los países europeos entienden el pensamiento computacional de la misma manera (Bocconi et al., 2016).

En este informe se distinguen los países con políticas nacionales de los países con políticas a nivel regional. En este sentido, dentro de los países con políticas a nivel nacional, distingue tres grupos de países en función del enfoque que han adoptado para introducir el pensamiento computacional y la computación en la enseñanza obligatoria (Bocconi et al., 2016). El primer grupo está compuesto por países que en los últimos seis años han revisado o están revisando sus currículos. En todos los países de este primer grupo, se está impulsando la enseñanza de pensamiento computacional y términos relacionados con el pensamiento computacional, en la enseñanza obligatoria. En este primer grupo se encuentran Inglaterra, Francia, Portugal, Finlandia, Polonia, Italia, Dinamarca, Malta, Croacia y Escocia (Bocconi et al., 2016). El segundo grupo está conformado por países que todavía no han incluido el pensamiento computacional en el currículo, pero tienen planes para hacerlo. En este grupo se encuentran República Checa, Irlanda, Noruega, Gales, Grecia, Países Bajos y Suecia (Bocconi et al., 2016). El tercer grupo está formado por países con una dilatada trayectoria en pensamiento computacional, En este grupo se incluyen Austria, Chipre, Israel, Lituania, Hungría y Eslovaquia (Bocconi et al., 2016).

En los países en los que el currículo se desarrolla a nivel regional, la integración del pensamiento computacional es distinta de una región a otra. En el caso de España, Andalucía, Castilla-La Mancha, Castilla y León, Galicia, Región de Murcia y Comunidad Valenciana han introducido nuevas asignaturas de robótica y programación en Secundaria (INTEF, 2018). Por otro lado, la Comunidad de Madrid y Cataluña lo han hecho tanto en primaria como en secundaria. Cantabria, La Rioja y las Islas Baleares ni han introducido asignaturas ni han creado contenidos en asignaturas ya existentes (INTEF, 2018). En este sentido, Navarra ha introducido contenidos de pensamiento computacional en la materia de matemáticas de educación primaria (Código 21, 2015).

En otros países europeos también existen iniciativas a nivel regional en aquellos en los que el currículo se desarrolla a nivel regional. En Alemania existen iniciativas en Baviera y Renania del Norte-Westfalia. En las regiones flamencas de Bélgica los alumnos de los grados 11 y 12 tienen perfiles de estudio que incluyen contenidos de pensamiento computacional. Estos perfiles forman parte de los estudios de Economía y Administración. En las zonas de habla alemana de Suiza, se incluyen dos competencias del pensamiento computacional (codificación y programación) en primaria y secundaria. En la parte de habla francesa de Suiza el pensamiento computacional forma parte del MITIC (Medios, Imagen y TIC) (Bocconi et al., 2016). 


\section{Discusión y Conclusiones}

El objetivo de este trabajo fue conocer los beneficios que el pensamiento computacional aporta a la educación y las propuestas de aplicación del mismo en Educación Secundaria tanto en las distintas regiones españolas como en otros países europeos. A lo largo del presenta trabajo se ha descrito el concepto de pensamiento computacional, la tendencia actual en muchos países a introducirlo en el currículo, así como una serie de habilidades, características y actitudes asociadas a él, entre las que destacan la abstracción (Angeli et al., 2016; Barr y Stephenson, 2011; Grover y Pea, 2013; Lee et al., 2011; Selby y Woollard, 2013; Wing, 2011), la descomposición de problemas (Angeli et al., 2016; Barr y Stephenson, 2011; Grover y Pea, 2013; Selby y Woollard, 2013; Wing, 2006), el pensamiento algorítmico (Angeli et al., 2016; Barr y Stephenson, 2011; Grover y Pea, 2013; Selby y Woollard, 2013; Wing, 2011), la automatización (Barr y Stephenson, 2011; Lee et al., 2011; Wing, 2008), la depuración de errores (Angeli et al., 2016; Grover y Pea, 2013) y la generalización (Angeli et al., 2016; Selby y Woollard, 2013; Wing, 2011).

De igual manera, basándose en la bibliografía consultada, se han fundamentado los beneficios que el pensamiento computacional aporta a la educación: mejora de la competencia digital, desarrollo y fortalecimiento de habilidades socio-emocionales, habilidades relacionadas con el lenguaje y la comunicación y potenciación del trabajo colectivo (Gurises Unidos, 2017). Asimismo, permite mejorar la competencia del sentido de la iniciativa y espíritu emprendedor, ya que fomenta el liderazgo, la innovación y el emprendimiento (Posada, 2017). Igualmente, se ha fundamentado el pensamiento computacional como una de las maneras de enfocar el aprendizaje basado en problemas (Gurises Unidos, 2017; Lee et al., 2011).

Por otro lado, se han descrito las distintas iniciativas en Europa y en las Comunidades Autónomas de España a la hora de introducir el pensamiento computacional en el currículo. En este sentido, en Inglaterra, Francia, Portugal, Finlandia, Polonia, Italia, Dinamarca, Malta, Croacia y Escocia se está impulsando la enseñanza de pensamiento computacional y términos relacionados con el pensamiento computacional, en la enseñanza obligatoria (Bocconi et al., 2016). Por otro lado, en República Checa, Irlanda, Noruega, Gales, Grecia, Países Bajos y Suecia no han incluido el pensamiento computacional en el currículo, pero tienen planes para hacerlo (Bocconi et al., 2016). En Austria, Chipre, Israel, Lituania, Hungría y Eslovaquia existe una amplia trayectoria de pensamiento computacional en el currículo (Bocconi et al., 2016). En cuanto a España, Andalucía, Castilla-La Mancha, Castilla y León, Galicia, Región de Murcia y Comunidad Valenciana han introducido nuevas asignaturas de robótica y programación en Secundaria (INTEF, 2018). Asimismo, la Comunidad de Madrid y Cataluña lo han hecho tanto en primaria como en secundaria. Cantabria, La Rioja y las Islas Baleares ni han introducido asignaturas ni han creado contenidos en asignaturas ya existentes (INTEF, 2018). Por último, Navarra ha introducido contenidos de pensamiento computacional en la materia de matemáticas de educación primaria (Código 21, 2015).

Las principales limitaciones que presenta este estudio están relacionadas con la complejidad para generalizar los beneficios del pensamiento computacional, dada 
la multitud de definiciones y cantidad de términos asociados al mismo. De igual manera, no todos los países entienden el pensamiento computacional de la misma forma, lo que dificulta identificar las iniciativas de pensamiento computacional en los distintos currículos de secundaria. Por otra parte, únicamente se ha realizado la búsqueda usando términos en inglés y castellano, sin tener en cuenta bibliografía publicada en otros idiomas. Por último, otra dificultad fue la imposibilidad de comprar el pensamiento computacional en otros países en lengua española, como pueden ser los países iberoamericanos, debido a que el objetivo principal de la investigación fue examinar las iniciativas en España y Europa, y por ello, el trabajo se centra sólo en España y el territorio europeo.

En cuanto a posibles líneas de futuro, sería interesante ampliar la búsqueda a otros idiomas. De igual manera sería interesante ampliar el estudio al currículo de secundaria de otros países de fuera de Europa. Por otro lado, dado que existen países que tienen planes de introducción del pensamiento computacional en el currículo, sería interesante estudiar cómo van implantando estos planes. Por último, debido a que el Ministerio de Educación y Formación Profesional está potenciando la introducción del pensamiento computacional en las aulas a través de un proyecto de escuela de pensamiento computacional, sería interesante revisar cómo las distintas Comunidades Autónomas españolas están avanzando en la introducción del pensamiento computacional en las aulas de secundaria.

Como conclusiones finales del presente trabajo de revisión narrativa, se destacan las siguientes:

- Destaca la tendencia actual en muchos países a introducir en el currículo el pensamiento computacional, así como una serie de habilidades, características y actitudes asociadas a él, entre las que destacan: la abstracción, la descomposición de problemas, el pensamiento algorítmico, la automatización, la depuración de errores y la generalización.

- Los principales beneficios que aporta el pensamiento computacional son: mejora de la competencia digital, desarrollo y fortalecimiento de habilidades socio-emocionales, habilidades relacionadas con el lenguaje y la comunicación y potenciación del trabajo colectivo.

- La mayoría de países Europeos ha implementado o está en vías de implementar más acciones para fortalecer el uso del pensamiento computacional entre el alumnado.

\section{REFERENCIAS BIBLIOGRÁFICAS}

Allan, W., Coulter, B., Denner, J., Erickson, J., Lee, I., Malyn-Smith, J. y Martin, F. (2010). Computational Thinking for Youth. ITEST Small Working Group on Computational Thinking.

Angeli, C., Voogt, J., Fluck, A., Webb, M., Cox, M., Malyn-Smith, J., y Zagami, J. (2016). A K-6 Computational Thinking Curriculum Framework- Implications for Teacher Knowledge. Educational Technology y Society, 19(3), 47-57. 
Barr, D., Harrison, J. y Conery, L. (2011). Computational Thinking: A Digital Age Skill for Everyone. Learning y Leading with Technology, 38(6), 20-23.

Barr, V. y Stephenson, C. (2011). Bringing Computational Thinking to K-12: What is Involved and What is the Role of the Computer Science Education Community? ACM Inroads, 2(1), 48-54.

Basogain Olabe, X., Olabe Basogain, M. A. y Olabe Basogain, J. C. (2015). Pensamiento Computacional a través de la Programación: Paradigma de Aprendizaje. Revista De Educación a Distancia, 46(6). http://doi.org/10.6018/red/46/6

Bocconi, S., Chioccariello, A., Dettori, G., Ferrari, A. y Engelhardt, K. (2016). Developing computational thinking in compulsory education - Implications for policy and practice. Luxembourg: Publications Office of the European Union. Recuperado de https://ec.europa.eu/jrc/en/publication/eur-scientific-and-technical-researchreports/developing-computational-thinking-compulsory-education-implicationspolicy-and-practice

Caballero-González, Y. A. y García-Valcárcel, A. (2019). ¿Aprender con robótica en Educación Primaria? Un medio de estimular el pensamiento computacional. Education in the Knowledge Society, 20(1), 1-15. http://doi.org/10.14201/eks.21443

Código 21 (2015). Programar para aprender: orientaciones para el profesorado de Primaria. Pamplona: Gobierno de Navarra, Departamento de Educación. Recuperado de http://codigo21.educacion.navarra.es/profesorado/guia-programar-paraaprender-orientaciones-para-el-profesorado-de-primaria/

CSTA e ISTE (2011). Operational definition of Computational Thinking in K-12 education. Recuperado de https://id.iste.org/docs/ct-documents/computational-thinking-operational-definition-flyer.pdf?sfvrsn=2

Grover, S. (2018). The 5th ' $\mathrm{C}$ ' of 21st Century Skills? Try Computational Thinking (Not Coding). Edsurge. Recuperado de https://www.edsurge.com/news/201802-25-the-5th-c-of-21 st-century-skills-try-computational-thinking-not-coding.

Grover, S. y Pea, R. (2013). Computational Thinking in K-12 A Review of the State of the Field. Educational Researcher, 42(1), 38-43. https://doi. org/10.3102/0013189X12463051

Gurises Unidos (2017). Pensamiento computacional. Un aporte para la educación de hoy. Montevideo, Uruguay: Gurises Unidos. Recuperado de https://www.gurisesunidos.org.uy/wp-content/uploads/2017/11/PensamientoComputacional.pdf.

INTEF, Instituto Nacional de Tecnologías Educativas y de Formación del Profesorado (2018). Programación, robótica y pensamiento computacional en el aula. Situación en España. Madrid: Ministerio de Educación y Formación Profesional, INTEF. Recuperado de https://code.intef.es/wp-content/uploads/2018/10/Ponenciasobre-Pensamiento-Computacional.-Informe-Final.pdf

INTEF, Instituto Nacional de Tecnologías Educativas y de Formación del Profesorado (2018). Situación de la competencia digital del alumnado en España - Comparati- 
va de iniciativas. INTEF. Recuperado de https://intef.es/formacion-y-colaboracion/ competencia-digital-educativa/

Lamprou, A. y Repenning, A. (2018). Computational Thinking $\neq$ Programming. SI Digital Magazine (SIDM). Recuperado de https://magazine.swissinformatics.org/en/ computational-thinking-\#-programming/\#_ftn1

Lee, I., Martin, F., Denner, J., Coulter, B., Allan, W., Erickson, J., Malyn-Smith, J. y Werner, L. (2011). Computational thinking for youth in practice. ACM Inroads, 2(1), 32-37. https://doi.org/10.1145/1929887.1929902

Posada, F. (2017). Pensamiento computacional en el aula. Póster presentado en Reunión Coordinadores TIC, Lanzarote, noviembre 2017.

Real Decreto 1105/2014, de 26 de diciembre, por el que se establece el currículo básico de la Educación Secundaria Obligatoria y del Bachillerato. Boletín Oficial del Estado, 3, de 3 de enero de 2015.

Roig-Vila, R. y Moreno-Isac, V. (2020). El pensamiento computacional en educación. Análisis bibliométrico y temático. RED. Revista de Educación a Distancia, 63(20), 1-24. http://doi.org/10.6018/red.402621

Román, M., Pérez, J. C. y Jiménez, C. (2015). Test de Pensamiento Computacional: diseño y psicometría general. En III Congreso Internacional sobre Aprendizaje, Innovación y Competitividad, Madrid, octubre 2015.

Sánchez Palacios, M. C. (2018). Enseñar Economía mediante el ABP: aprendizaje basado en problemas en Bachiller. Campus Educación Revista Digital Docente 8, 47-50. Recuperado de https://www.campuseducacion.com/revista-digitaldocente/numeros/8

Selby, C. C. y Woollard, J. (2013). Computational Thinking: The Developing Definition. University of Southampton (E-prints) 6 pp. Recuperado de https://eprints. soton.ac.uk/356481/

The Royal Society (2012). Shut down or restart? The way forward for computing in UK schools. Recuperado de https://royalsociety.org/ /media/education/computing-inschools/2012-01-12-computing-in-schools.pdf

Weintrop, D., Beheshti, E., Horn, M., Orton, K., Jona, K., Trouille, L. y Wilensky, U. (2016). Defining Computational Thinking for Mathematics and Science Classrooms. Journal of Science Education and Technology, 25, 127-147. https:// doi.org/10.1007/s10956-015-9581-5

Wing, J. M. (2006). Computational thinking. Communications of the ACM, 49(3), 3335. https://doi.org/10.1145/1118178.1118215

Wing, J. M. (2008). Computational thinking and thinking about computing. Philosophical Transactions of the Royal Society of London A: Mathematical, Physical and Engineering Sciences, 366(1881), 3717-3725. https://doi.org/10.1098/ rsta.2008.0118 
Wing, J. M. (2011). Research Notebook: Computational Thinking-What and Why? The Link, The magazine of Carnegie Mellon University's School of Computer Science. Recuperado de https://www.cs.cmu.edu/link/research-notebook-computationalthinking-what-and-why

Woollard, J. (2016). CT Driving Computing Curriculum in England. CSTA Voice, 12(1), 4-5.

Zapata-Ross, M. (2015). Pensamiento computacional: Una nueva alfabetización digital. RED. Revista de Educación a Distancia, 46, 47 pp. Recuperado de https:// revistas.um.es/red/article/view/240321 